\title{
Do Boys and Girls Behave Differently Online?
}

\section{A Review of Gender Differences in Cyberbullying}

\author{
Yuanyuan Chang $^{1 *}$ \\ ${ }^{1}$ College of William and Mary \\ "Corresponding author. Email: ychang03@email.wm.edu
}

\begin{abstract}
As more and more adolescents gain access to electronic devices and the Internet, cyberbullying has become a widespread issue. One disputable aspect of cyberbullying is whether there are gender differences among cyberbullies and cybervictims. The current review analyzed previous studies on gender patterns in cyberbullying among teenagers and compare the results with that of traditional bullying. The review shows that research on the gender differences of cyberbullying has yielded mixed results. Although some studies find gender differences similar to that found in traditional bullying, with boys more likely to be cyberbullies while girls are more often victimized, many studies show no significant differences while others find alternative patterns. Several factors may play a role in varying the results, for instance, the inconsistent definition and measurement implemented in the studies, the interaction between age and gender, and differential gender norms resulted from distinct cultural backgrounds. Investigating the gender differences in cyberbullying may give insights into the differential socialization of either gender and help improve cyberbullying prevention programs. Therefore, to continue such investigation, future studies are advised to develop a more consistent measure of cyberbullying behaviors, to analyze further the effect of culture and age, and to take into consideration nonbinary gender identities.
\end{abstract}

Keywords: cyberbullying, gender, adolescence

\section{INTRODUCTION}

With swift advances in technologies, access to the Internet and electronic devices has become more and more ubiquitous among adolescents. However, time spent in the anonymous digital world is not always pleasant. With common Internet use, cyberbullying also prevails among adolescents. Cyberbullying is defined as intentional acts of aggression mediated through electronic devices, repeatedly or over time against a victim who is in a disadvantageous in defending oneself [1]. Examples of cyberbullying includes, and is not limited to, sending nasty, name-calling or threatening text message to someone, hacking or faking others' social media accounts, and ignoring, defriending or excluding someone on social media or online games [2]. Cyberbullying has been linked to several unfavorable conditions, both physical and psychological [3]. Meanwhile, gender differences in cyberbullying remain disputable.
Gender patterns in cyberbullying have generated mixed results [4], [5]. Despite the abundance of data available regarding the gender differences, little research actually focuses on addressing this result [6], [7]. Gender patterns in cyberbullying prevalence and specific aggression/victimization behaviors may shine some light on the development of intervention and prevention programs [8]. More importantly, cyberbullying, whether being the cybervictim or the cyberbully, is an important aspect of adolescents' social behaviors. Looking into the gender differences in cyberbullying may allow us to gain some insights on how these youngsters are socialized differentially based on their gender and how they perform their gender identity through such social behaviors [9]. Therefore, the current review discusses the gender differences among cyberbullies and cybervictims and compare the differences between traditional bullying and cyberbullying in terms of gender patterns. 


\section{GENDER DIFFERENCES IN CYBERVICTIMS AND CYBERBULLIES}

Gender differences in cyberbullying have yielded mixed results. In regard to cyberbullying victimization, some research shows that adolescent girls are more often the subject of cyberbullying [10], [11], while others find no significant gender difference [12], [13]. Fanti and Colleagues [14] found boys more likely to be victimized online than girls. As for cyberbullies, several studies conclude that boys are more likely to be the cyberbully [11], [14]-[16]. In contrast, other research finds either gender equally likely to be bullies on the Internet [10], [17], [18]. Most research in the field investigates gender differences with a binary definition of gender-male or female. Among the few studies that discuss adolescents with non-binary gender identities, one study found nonbinary adolescents at higher risks of victimization than their cisgender peers [19]. Although studies with significant results conform to the gender stereotypes that males are more aggressive and females are more vulnerable, due to the abundance of studies showing nonsignificant data, it remains inconclusive whether gender differences actually exist in cyberbullying.

The studies are conducted under different cultures and nationalities. The majority are conducted in North America and Europe [12], [17], [18], [20]. Noticeably, even though participants in these studies are from similar western backgrounds, these studies have yielded conflicting results. Similar inconsistency has also been found in other continents: studies conducted in Asia found males more prevalent in cyberbullying in Hongkong, Taiwan, and Mainland China [21], while research in Turkey and South Korea showed no gender difference in both cyberbullies and cybervictims [22], [23]. In Australia, Perren and colleagues [15] found boys more likely to be cyberbullies while other research with Australian teenagers found either gender equally prone to cyberbullying [24], [25]. Moreover, studies investigating gender patterns in cyberbullying have adopted various definitions of cyberbullying. Some research stressed the aggressiveness, repetitiveness, and intentionality of the behavior [12], [14], [18], while others, usually earlier ones, simply defined cyberbullying as bullying involving electronic devices [9], [15], [17]. The majority of the studies employed questionnaires to measure cyberbullying, nevertheless, with different definitions, the measuring scales also varied. Some studies gave out a definition or a description of cyberbullying and asked directly if the participant has perpetuated or received such behaviors [5], [12], whereas others surveyed about specific cyberbullying actions [14], [15], [17], [18]. Commonly measured actions are sending nasty/threatening messages via email or text [14], [15], [17] and leaving mean comments on social media or websites [14], [15]. Besides these verbal aggressions, some research also investigated more indirect actions of cyberbullying, such as the exclusion of online companies and the impersonation or hacking of one's online [18].

\section{GENDER DIFFERENCES IN TRADITIONAL BULLYING: IN COMPARISON WITH CYBERBULLYING}

Research on cyberbullying are largely guided by that of traditional bullying [26]. While the debate of gender differences in cyberbullying is ongoing, studies of such patterns in traditional bullying are less disputable. It is worth comparing their respective research in gender differences since results from traditional bullying may lead the investigation in cyberbullying. Traditional bullying is defined as peer victimization characterized by three key components: ill intentionality, repeated perpetuation and disproportion of strength between the victim and the bully [27]. Various literature also identifies these characteristics as key parts of cyberbullying [1], [26]. Examples of traditional bullying behaviors are physical aggression such as kicking, beating, or pushing someone, often a peer, damaging or occupying one's property, and embarrassing, insulting, or name-calling someone [2]. Gender patterns in traditional bullying have been widely found: Overall, boys are more likely to bully others than girls [5], [28]. In terms of victimization, most studies show that teenage girls are more likely to be victimized than boys [28], others report no significant differences [5], [15]. In comparison, as illustrated above, though some studies in cyberbullying have found similar patterns, many find such gender differences insignificant. A few studies even found differential results, with boys more likely to be both the cyberbully and the cybervictim [14], [29].

\section{DISCUSSION}

Overall, the current review finds studies on the gender differences in cyberbullying generating heterogeneous and therefore inconclusive results. In comparison, studies on traditional bullying have established that teenage boys are more likely to be bullies than girls, and that teenage girls are more often the victims. Although some studies on cyberbullying find gender patterns consistent with that of traditional bullying, many show no gender difference or find alternative patterns.

Several factors may play a role in diversifying the results in cyberbullying. Firstly, as discussed earlier, studies have adopted various definitions of cyberbullying and have used different questionnaire items as measurements, with some measured verbal aggression and others included relational aggression [15], [17], [18]. Results from traditional bullying have shown that boys are more likely to be involved in physical and verbal forms of bullying and less likely to be involved in relational bullying [30]. In the case of cyberbullying, it is plausible that such pattern persists, therefore, studies that do not include relational forms of cyberbullying may 
skew their results toward males. Another potential confounding factor is age. Although this review is dedicated to the analysis of cyberbullying among adolescents, the studies still have participants of varying age ranges. For instance, Fanti and colleagues [14] surveyed participants in their early adolescence, aged from 11 to 14 years. In comparison, Slonje and Smith [13] incorporated an ampler range of adolescents, sampling participants from 12 to 20 years old. Age as a confounding factor can potentially skew the results as the interaction effect between age and gender have been found in cyberbullying. Baker and Tanrikulu [22] found that gender together with age is related to being a cyberbully but not a cybervictim. Specifically, 14-yearold female students were found significantly more likely to be cyberbullies than their younger peers, while boys of different ages did not differ. C. Barlett and Coyne [16] have concluded that age is a moderator for gender's effect on being a cyberbully: More females report to be cyberbullies in their early adolescents, and more males are cyberbullies in late adolescence. On top of that, Björkqvist and colleges [31] have found that prevalence of indirect aggression in girls grows larger as participants' age increases, suggesting that the use of indirect aggression is dependent on teenagers' maturation and the formation of their social network, with which relational bullying is made possible. It is possible that in the case of cyberbullying, because females mature faster verbally and socially, they are capable of utilizing verbal (nasty text message) and relational means (defriending on social media) to bully others on the Internet earlier than males are, resulting in discrepant gender patterns of cyberbullying in different age groups. Last but not least, culture plays an important role in the gender differences of cyberbullying. The analysis by $\mathrm{C}$. Barlett and Coyne [16] has shown that gender differences in cyberbullying are found in North America, Europe, and Asia, with Asia having the largest effect size, and not for Australia. Social norms for both genders are determined by one's culture, and it is likely that aggressiveness of the male is more socially approved in some regions of the world than the others, resulting in larger gender differences in cyberbullying.

Gender patterns have been found more consistently in traditional bullying than in cyberbullying, and this discrepancy could be accounted for by the distinct characteristics of the two phenomena. Despite commonalities, traditional bullying and cyberbullying are different in several aspects [26]: anonymity, one crucial risk factor for cyberbullying behavior [32], [33]; accessibility of the victims [34]; and presence of intervention [26]. All these factors could play a role in skewing the gender prevalence. Take anonymity as an example: In patriarchal societies, masculine features such as pugnacity and dominance are regarded as favorable for males, whereas conventionally feminine features such as submissiveness and tenderness are cultivated in females
[35]. As a result, aggressive behaviors like bullying are more socially acceptable when done by males as compared to by females. The double standard of social desirability may partially account for the pattern that boys are more likely to bully others. Nevertheless, in the anonymous cyber world, an online account can provide invisibility of identity, with which also comes the invisibility of gender identity. Without the burden of social expectations for their gender, it is possible that girls are less refrained from deviant behaviors such as cyberbullying, rebalancing the gender differences among the cyberbullies. Moreover, traditional bullying behaviors can be categorized as physical (e.g., beating someone), verbal (e.g. name-calling), and relational (e.g. spreading rumors or excluding someone from a social group) [36], [37]. Relational bullying is also referred to as the indirect form of aggression, as opposed to verbal and physical bullying as the direct form [38]. The overall gender pattern found in traditional bullying conforms to the social norms that male is the more aggressive sex, nonetheless, research reveals that both sexes have their aggressive side, only that they take different forms. Studies have supported that boys are more likely to engage in physical and verbal aggression, whereas girls more often perpetuate relational bullying or indirect forms of aggression [31], [38]. On the other hand, the nature of cyberbullying determines that it cannot fit into this categorization, as physical aggression cannot be mediated through the Internet. Since there are gender differences within the sub-categories of traditional bullying, it is likely that the lack of a physical component of cyberbullying accounts for the discrepancy of gender differences in the two phenomena.

Studies concerning gender differences in cyberbullying have several limitations. Firstly, the term "cyberbullying" has been used inconsistently. With varying definitions of cyberbullying, research in the field has employed different approaches to measuring the phenomena, potentially complicating the results. Future research should aim at composing a consensual definition of cyberbullying as well as a reliable, comprehensive measurement for it. Secondly, as mentioned earlier, several factors may serve as confounding variables in the research, among which are age and culture. Although the current review investigates specifically cyberbullying among adolescents, study shows that within this period of rapid development, even an age difference of one year changes the prevalence of cyberbullying [22]. Future studies should pay attention to teasing apart the effect of age on cyberbullying and the interaction between age and gender. To do so, a more specific age grouping is necessary. Moreover, as most of the studies in the field are cross-sectional (e.g., [21], [39]), research in the future should consider conducting more longitudinal studies in order to better grasp the dynamic relationship between gender, age and cyberbullying. Similarly, the role of culture requires more detailed investigations. Previous 
research has analyzed the cultural difference in the gender patterns among different continents [16], however, as this review has shown, national results within one continent also vary. Therefore, future investigations are advised to analyze gender differences at a national level, taking into account not only cultural but also political and economic differences of different countries. Cultural differences in the gender patterns of cyberbullying lack a plausible explanation. Culture is an umbrella term that includes the beliefs, customs, habits, and language of a group of people [40]. Within culture, numerous factors could affect the gender differences in cyberbullying. For instance, western culture may have traditionally cultivated distinct qualities in males and females, nonetheless, there are also currents of political movements against this gender stereotyping. Culture is a multifaceted factor that incorporates multiple variables. Future studies should aim at teasing out from "culture" more specific factors accountable for the differential gender patterns. Results from less investigated countries and those removed from mainstream culture can be exceptionally valuable since it may allow for clear comparisons with the mainstream results. Last but not least, the majority of the studies reviewed have employed a binary categorization of gender [12], [24]. Although some included a third category [18], very few incorporated non-binary gender identities. Studies taking into account non-binary genders would be a beneficial supplement to this topic. Moreover, some studies used the term sex [11]; some used gender [12], [15], and a few used the two terms interchangeably [10]. On top of that, it is unknown which term and what phrasing were used to ask the participants their gender or sex. The two terms are different concepts, and non-cisgender participants may answer differently depending on the phrasing of the questions. Future studies are advised to be more cautious with the use of the two terms, choosing based on whether the research question is more concerned with the biological aspect or with the social aspect of cyberbullying.

Though the previous studies have several limitations, they have shown practical implications. Results on gender differences in cyberbullying may help improve the development of cyberbullying intervention and prevention programs. For instance, having found the three-way interaction between gender, affective empathy, and cognitive empathy on cyberbullying, Ang and Goh [8] proposed an empathy training with an emphasis on cognitive components of empathy for boys and affective components of empathy for girls as a supplement for cyberbullying intervention programs. Future research may further investigate factors that interact with gender so that prevention of cyberbullying can be tailored for either gender according to their respective characteristics.

\section{CONCLUSION}

In conclusion, the current review finds mixed results in previous studies on the gender differences of cyberbullying among adolescents. In contrast, traditional bullying has yielded more consistent results, with boys more likely to be the bully and girls more likely to be the victim. This discrepancy may have to do with the distinctiveness of the cyberspace and the differential categorizations of the two phenomena. Analyzing gender differences in cyberbullying may improve cyberbullying prevention by crafting intervention programs differently for either gender. Current research on the topic still has several limitations, and future studies are advised to reach a more consistent definition of cyberbullying, examine the influences of age and culture in more detail, and use more caution with the wording and categorization of gender.

\section{REFERENCES}

[1] P. K. Smith, J. Mahdavi, M. Carvalho, S. Fisher, S. Russell, and N. Tippett, "Cyberbullying: its nature and impact in secondary school pupils," J. Child Psychol. Psychiatry, vol. 49, no. 4, pp. 376-385, Apr. 2008, doi: 10.1111/j.1469-7610.2007.01846.x.

[2] S. R. Sumter, P. M. Valkenburg, S. E. Baumgartner, J. Peter, and S. van der Hof, "Development and validation of the Multidimensional Offline and Online Peer Victimization Scale," Comput. Human Behav., vol. 46, pp. 114-122, 2015, doi: https://doi.org/10.1016/j.chb.2014.12.042.

[3] R. M. Kowalski and S. P. Limber, "Psychological, Physical, and Academic Correlates of Cyberbullying and Traditional Bullying," J. Adolesc. Heal., vol. 53, no. 1, Supplement, pp. S13S20, 2013, doi: https://doi.org/10.1016/j.jadohealth.2012.09.018.

[4] J. Wang, T. R. Nansel, and R. J. Iannotti, “Cyber and Traditional Bullying: Differential Association With Depression," J. Adolesc. Heal., vol. 48, no. 4, pp. 415-417, 2011, doi: https://doi.org/10.1016/j.jadohealth.2010.07.012.

[5] L. Beckman, C. Hagquist, and L. Hellström, "Discrepant gender patterns for cyberbullying and traditional bullying - An analysis of Swedish adolescent data," Comput. Human Behav., vol. 29, no. 5, pp. 1896-1903, Sep. 2013, doi: 10.1016/j.chb.2013.03.010.

[6] D. A. Cole et al., "Longitudinal and Incremental Relation of Cybervictimization to Negative SelfCognitions and Depressive Symptoms in Young Adolescents," J. Abnorm. Child Psychol., vol. 44, no. 7, pp. 1321-1332, 2016, doi: 10.1007/s10802015-0123-7. 
[7] V. A. Coelho and A. M. Romão, "The relation between social anxiety, social withdrawal and (cyber)bullying roles: A multilevel analysis," Comput. Human Behav., vol. 86, pp. 218-226, 2018 , doi: https://doi.org/10.1016/j.chb.2018.04.048.

[8] R. P. Ang and D. H. Goh, "Cyberbullying among adolescents: The role of affective and cognitive empathy, and gender," Child Psychiatry Hum. Dev., vol. 41, no. 4, pp. 387-397, 2010, doi: 10.1007/s10578-010-0176-3.

[9] R. Navarro, S. Yubero, E. Larrañaga, and V. Martínez, "Children's cyberbullying victimization: Associations with social anxiety and social competence in a Spanish sample," Child Indic. Res., vol. 5, no. 2, pp. 281-295, 2012, doi: $10.1007 / \mathrm{s} 12187-011-9132-4$.

[10] L. Beckman, C. Hagquist, and L. Hellström, "Discrepant gender patterns for cyberbullyiyng and traditional bullying - An analysis of Swedish adolescent data," Comput. Human Behav., vol. 29, no. 5, pp. 1896-1903, 2013, doi: 10.1016/j.chb.2013.03.010.

[11] S. Wachs, M. Junger, and R. Sittichai, "Traditional, Cyber and Combined Bullying Roles: Differences in Risky Online and Offline Activities," Societies, vol. 5, no. 1, pp. 109-135, 2015, doi: $10.3390 /$ soc5010109.

[12] K. Athanasiou et al., "Cross-national aspects of cyberbullying victimization among 14-17-year-old adolescents across seven European countries," BMC Public Health, vol. 18, no. 1, 2018, doi: 10.1186/s12889-018-5682-4.

[13] R. Slonje and P. K. Smith, "Cyberbullying: Another main type of bullying?," Scand. J. Psychol., vol. 49, no. 2, pp. 147-154, Apr. 2008, doi: 10.1111/j.14679450.2007.00611.x.

[14] K. A. Fanti, A. G. Demetriou, and V. V. Hawa, “A longitudinal study of cyberbullying: Examining risk and protective factors," Eur. J. Dev. Psychol., vol. 9, no. 2, pp. 168-181, 2012, doi: 10.1080/17405629.2011.643169.

[15] S. Perren, J. Dooley, T. Shaw, and D. Cross, "Bullying in school and cyberspace: Associations with depressive symptoms in Swiss and Australian adolescents," Child Adolesc. Psychiatry Ment. Health, vol. 4, pp. 1-10, 2010, doi: 10.1186/17532000-4-28.

[16] C. Barlett and S. M. Coyne, "A meta-analysis of sex differences in cyber-bullying behavior: The moderating role of age," Aggress. Behav., vol. 40, no. 5, pp. 474-488, Sep. 2014, doi: 10.1002/ab.21555.

[17] K. R. Williams and N. G. Guerra, "Prevalence and Predictors of Internet Bullying," J. Adolesc. Heal., vol. 41, no. 6 SUPPL., pp. 14-21, 2007, doi: 10.1016/j.jadohealth.2007.08.018.

[18] E. Calvete, I. Orue, A. Estévez, L. Villardón, and P. Padilla, "Cyberbullying in adolescents: Modalities and aggressors' profile," Comput. Human Behav., vol. 26, no. 5, pp. 1128-1135, 2010, doi: 10.1016/j.chb.2010.03.017.

[19] M. E. Aparicio-García, E. M. Díaz-Ramiro, S. Rubio-Valdehita, M. I. López-Núñez, and I. GarcíaNieto, "Health and well-being of cisgender, transgender and non-binary young people," Int. J. Environ. Res. Public Health, vol. 15, no. 10, 2018, doi: 10.3390/ijerph15102133.

[20] L. Beckman, C. Hagquist, and L. Hellström, "Does the association with psychosomatic health problems differ between cyberbullying and traditional bullying?," Emot. Behav. Difficulties, vol. 17, no. 3-4, pp. 421-434, Sep. 2012, doi: 10.1080/13632752.2012.704228.

[21] J.-K. Chen and L.-M. Chen, "Cyberbullying among adolescents in Taiwan, Hong Kong, and Mainland China: a cross-national study in Chinese societies," Asia Pacific J. Soc. Work Dev., vol. 30, no. 3, pp. 227-241, Jul. 2020, doi: 10.1080/02185385.2020.1788978.

[22] Ö. E. Baker and I. Tanrikulu, "Psychological consequences of cyber bullying experiences among Turkish secondary school children," Procedia - Soc. Behav. Sci., vol. 2, no. 2, pp. 2771-2776, 2010, doi: 10.1016/j.sbspro.2010.03.413

[23] S. Park, E. Y. Na, and E. mee Kim, "The relationship between online activities, netiquette and cyberbullying," Child. Youth Serv. Rev., vol. 42, pp. 74-81, 2014, doi: 10.1016/j.childyouth.2014.04.002.

[24] S. A. Hemphill et al., "Longitudinal predictors of cyber and traditional bullying perpetration in Australian secondary school students," J. Adolesc. Heal., vol. 51, no. 1, pp. 59-65, 2012, doi: 10.1016/j.jadohealth.2011.11.019.

[25] L. Griezel, L. R. Finger, G. H. Bodkin-Andrews, R. G. Craven, and A. S. Yeung, "Uncovering the structure of and gender and developmental differences in cyber bullying," J. Educ. Res., vol. 105, no. 6, pp. 442-455, 2012, doi: 10.1080/00220671.2011.629692. 
[26] R. S. Tokunaga, "Following you home from school: A critical review and synthesis of research on cyberbullying victimization," Comput. Human Behav., vol. 26, no. 3, pp. 277-287, May 2010, doi: 10.1016/j.chb.2009.11.014.

[27] D. Olweus, "Bullying at School: Knowledge Base and an Effective Intervention Programa," Ann. N. Y. Acad. Sci., vol. 794, no. 1, pp. 265-276, 1996, doi: 10.1111/j.1749-6632.1996.tb32527.x.

[28] M. E. Solberg and D. Olweus, "Prevalence Estimation of School Bullying with the Olweus Bully/Victim Questionnaire," Aggress. Behav., vol. 29, no. 3, pp. 239-268, 2003, doi: 10.1002/ab.10047.

[29] W. V Pelfrey Jr and N. L. Weber, "Keyboard gangsters: Analysis of incidence and correlates of cyberbullying in a large urban student population," Deviant Behav., vol. 34, no. 1, pp. 68-84, 2013.

[30] J. Wang, R. J. Iannotti, and T. R. Nansel, "School Bullying Among Adolescents in the United States: Physical, Verbal, Relational, and Cyber," J. Adolesc. Heal., vol. 45, no. 4, pp. 368-375, 2009, doi: 10.1016/j.jadohealth.2009.03.021.

[31] K. Björkqvist, K. M. J. Lagerspetz, and A. Kaukiainen, "Do girls manipulate and boys fight? developmental trends in regard to direct and indirect aggression," Aggress. Behav., vol. 18, no. 2, pp. 117-127, 1992, doi: 10.1002/10982337(1992)18:2<117::AID-

AB2480180205>3.0.CO;2-3.

[32] C. P. Barlett, D. A. Gentile, and C. Chew, "Predicting cyberbullying from anonymity.," Psychol. Pop. Media Cult., vol. 5, no. 2, pp. 171180, Apr. 2016, doi: 10.1037/ppm0000055.

[33] M. J. Moore, T. Nakano, A. Enomoto, and T. Suda, "Anonymity and roles associated with aggressive posts in an online forum," Comput. Human Behav., vol. 28, no. 3, pp. 861-867, May 2012, doi: 10.1016/j.chb.2011.12.005.

[34] J. W. Patchin and S. Hinduja, "Bullies Move Beyond the Schoolyard," Youth Violence Juv. Justice, vol. 4, no. 2, pp. 148-169, Apr. 2006, doi: $10.1177 / 1541204006286288$.

[35] A. G. Johnson, "Patriarchy as a Personality Problem: Feminine and Masculine," in The Gender Knot: Unraveling Our Patriarchal Legacy, Temple University Press, 2005, pp. 85-90.

[36] D. Olweus, "Bullying at School BT - Aggressive Behavior: Current Perspectives,” L. R. Huesmann, Ed. Boston, MA: Springer US, 1994, pp. 97-130.
[37] N. R. Crick and J. K. Grotpeter, "Relational Aggression, Gender, and Social-Psychological Adjustment," Child Dev., vol. 66, no. 3, pp. 710 722, Jun. 1995, doi: 10.1111/j.14678624.1995.tb00900.x.

[38] L. Owens, R. Shute, and P. Slee, ?"'Guess what I just heard!?: Indirect aggression among teenage girls in Australia," Aggress. Behav., vol. 26, no. 1, pp. 6783, 2000, doi: 10.1002/(SICI)10982337(2000)26:1<67::AID-AB6>3.0.CO;2-C.

[39] J. Calmaestra, A. J. Rodríguez-Hidalgo, O. MeroDelgado, and E. Solera, "Cyberbullying in adolescents from Ecuador and Spain: Prevalence and differences in gender, school year and ethniccultural background," Sustain., vol. 12, no. 11, 2020, doi: 10.3390/su12114597.

[40] D. T. Kenrick, S. L. Neuberg, and R. B. Cialdini, Social Psychology: Goals in Interaction, 5th Editio. Pearson Education Limited, 2014. 